\title{
Adsorption Mechanisms of Microcystin Variant Conformations at Water-Mineral Interfaces: A Molecular Modeling Investigation
}

Amy L. Pochodylo, ${ }^{\dagger}$ Thalia G. Aoki, ${ }^{\ddagger}$ and Ludmilla Aristilde ${ }^{*},+\neq$

†The Institute for Comparative and Environmental Toxicology, College of Agriculture and Life Sciences, Cornell University, Ithaca, NY 14853 United States

‡Department of Biological and Environmental Engineering, College of Agriculture and Life Sciences, Cornell University, Ithaca, NY 14853 United States

${ }^{*}$ Corresponding Author:

Phone: (607) 255-6845. Fax: (607) 255-4449. E-mail: ludmilla@cornell.edu 


\section{ABSTRACT}

Microcystins (MCs) are potent toxins released during cyanobacterial blooms. Clay minerals are implicated in trapping MCs within soil particles in surface waters and sediments. In the absence of molecular characterization, the relevance of previously proposed adsorption mechanisms is lacking. Towards obtaining this characterization, we conducted Monte Carlo simulations combined with molecular dynamics relaxation of two MC variants, MC-leucine-arginine (MC-LR) and MC-leucine-alanine (MC-LA), adsorbed on hydrated montmorillonite with different electrolytes. The resulting adsorbate structures revealed how $\mathrm{MC}$ conformations and aqueous conditions dictate binding interactions at the mineral surface. Electrostatic coupling between the arginine residue and a carboxylate in MC-LR excluded the participation of arginine in mediating adsorption on montmorillonite in a $\mathrm{NaCl}$ solution. However, in a $\mathrm{CaCl}_{2}$ solution, the complexation of Ca by two carboxylate moieties in MC-LR changed the MC conformation, which allowed arginine to mediate electrostatic interaction with the mineral. By contrast, due to the lack of arginine in MC-LA, complexation of Ca by only one carboxylate in MC-LA was required to favor Ca-bridging interaction with the mineral. Multiple water-bridged $\mathrm{H}$-bonding interactions were also important in anchoring MCs at the mineral surface. Our modeling results offer molecular insights into the structural and chemical factors that can control the fate of MCs at water-mineral interfaces.

Keywords Adsorption Algal toxins Environmental partitioning 


\section{Graphical Abstract}
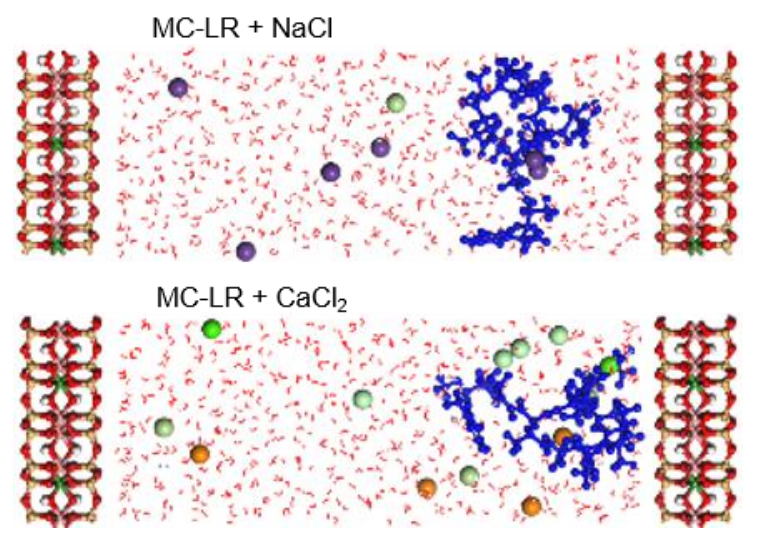


\section{Introduction}

Due to increasing nutrient loads, harmful algal blooms are proliferating in surface waters worldwide [1, 2]. Many cyanobacterial blooms release toxic compounds such as microcystins (MCs), which are potent liver toxins [1-4]. Due to their toxicity, lakes and rivers with high $\mathrm{MC}$ concentrations are not adequate for drinking water production or recreational use [5]. In addition, MCs are reported to be both toxic to fish and disruptive to aquatic food webs [6]. Therefore, understanding the mechanisms that control the fate of MCs is of great importance both to public and environmental health. In addition to biodegradation $[3-5,7-9]$ and photodegradation $[10,11]$, adsorption of MCs to particles in waters and sediments $[3,9,12-16]$ is considered to be one of the major processes responsible for the removal of MCs from contaminated surface waters. Of special interest is the elucidation of the mechanisms that drive the adsorption of different MC variants to minerals in suspended particles and sediments.

There are over 80 variants of MCs, which are monocyclic heptapeptides with two variable amino acids. The cyclic peptide in MCs contains a mixture of charged, polar, and nonpolar amino acid residues; all MCs also contain an Adda (3-amino-9-methoxy2,6,8-trimethyl-10-phenyldeca-4,6-dienoic acid) group attached to the peptide ring (Figure 1). The most toxic MC is MC-LR, which has a leucine (Leu) and an arginine (Arg) in the variable amino acid positions (Figure 1) [7]. Other common MCs include MC-RR with two Arg residues, MC-LW with a Leu and a tryptophan (Trp), and MC-LA with a Leu and an alanine (Ala) in the variable positions. Previous studies have reported of high retention of various MC variants in sediments enriched with clay content implied that clay minerals are important adsorbents of MCs $[14,16,17]$. In fact, because current 
cyanobacterial bloom treatments include the application of algaecides and chlorination, which can cause cell death and thus lead to the release of high levels of MCs [18-20], silicate minerals have been touted as efficient adsorbents for the removal of MCs [12, $21,22]$ and MC-containing cells $[20,23]$. The adsorption mechanisms have not yet been elucidated via spectroscopic or other molecular-scale techniques. However, previous macroscopic studies have led to several proposed mechanisms.

Previous adsorption studies $[13,15]$ have reported higher adsorption of MC-RR than MC-LR on natural sediments and the clay mineral montmorillonite. The positivelycharged guanidinium in the Arg residues is primarily implicated for the affinity of Argcontaining MCs for the negatively-charged mineral surfaces [15]. In addition to this electrostatic interaction, amino acid residues in the $\mathrm{MC}$ peptide ring are proposed to facilitate $\mathrm{H}$-bonding and cation-bridging interactions [12-15]. Moreover, electrolytes in solution are suggested to affect the conformation of MCs whereby changes in conformation seemed to be dependent on whether monovalent or divalent cations were in solution [24, 25]. However, the MC conformation required to enable favorable adsorption mechanisms under different aquatic chemistry conditions remains unknown.

Molecular modeling simulations have proven to be a powerful tool for obtaining complementary insights towards improving or adding to experimental interpretations of contaminant-clay interactions [26-32]. The objective of the present study is to employ this tool to elucidate the adsorbate structures and adsorption mechanisms responsible for the trapping of $\mathrm{MC}$ variants by clay minerals under different aquatic chemistry. The results from the aforementioned macroscopic adsorption studies have led us to the following hypotheses: (1) MC complexation with metal cations in the aqueous 
environment can induce specific MC conformation, (2) favorable MC conformation is required for optimal binding mechanisms to clay minerals, and (3) $\mathrm{H}$-bonding and metal complexation are the prevailing adsorption interactions for $\mathrm{MC}$ variants lacking a positive Arg residue. To evaluate these hypotheses, Monte Carlo simulations coupled with molecular dynamics relaxation were conducted to model the adsorption of two different MC variants, MC-LR and MC-LA (Figure 1), on montmorillonite. Montmorillonite (MONT) was chosen because it is an expandable clay mineral commonly found in soils and sediments. The simulations were performed in a fully-hydrated environment populated with either $\mathrm{NaCl}$ or $\mathrm{CaCl}_{2}$ in order to examine the effects of different electrolytes. The findings from this work provide mechanistic insights about the role of aquatic chemistry, $\mathrm{MC}$ chemistry, and MC conformation in the interaction of MCs with clay nanoparticles, thus allowing for better predictions of the potential of MCs to be trapped within soil particles in contaminated waters and sediments.

\section{Computational Methods}

\subsection{Modeling Platform}

Simulations were conducted using the all-atom Condensed-phase Optimized Molecular Potentials for Atomistic Simulation Studies (COMPASS; Accelrys, San Diego, CA, USA) [33] force field as implemented in the Materials Studio software package [34]. All atom descriptions including bonding and nonbonding terms were assigned by the COMPASS forcefield [33], except that partial charges for the water atoms were according to the extended simple point charge water model [35] and partial charges for the MONT atoms were according to the CLAYFF model [36]. The COMPASS force field 
$[35,39]$ previously demonstrated accurate simulations of structural properties of solvated metal cations [40], both simple and complex organic compounds [38-40], and adsorption of organic compounds to clay minerals [30, 32, 41]. We also performed additional validation simulations of MC-LR in order to ascertain that the COMPASS forcefield was adequate to model the adsorbates in our simulated systems. The Forcite module in Materials Studio was used to run geometry optimization, energy minimization, and molecular dynamics algorithms [34]. The Adsorption Locator module was used for conducting annealing Monte Carlo search of optimized adsorbate structures [34].

\subsection{Validation Studies}

We subjected hydrated MC-LR to a series of simulations consisting of geometry optimization, energy minimization (EM), and annealing molecular dynamics, as previously described $[38,39]$. Structural data were retrieved from a molecular dynamics production step which ran for $500 \mathrm{ps}$ (time step $=1 \mathrm{fs}$ ). This final molecular dynamics step was carried out in a canonical NVT ensemble (fixed number of atoms, simulation cell volume, and constant temperature) using a canonical Berendsen thermostat algorithm to control temperature at $298 \mathrm{~K}$. For all systems, the potential energy profile indicated that equilibrium was reached after 50 ps. Taking into account the structural flexibility of MC compounds, we also retrieved the root-mean-square-deviation (RMSD) profile of the MD-simulated MC-LR [Supporting Information (SI), Appendix A]. The RMSD profile reached toward an asymptotic slope after 50 ps, indicating that the total molecular dynamics time course of 500 ps was sufficient for obtaining optimized configurations of MC-LR. Bond lengths and bond angles were retrieved during the 
molecular dynamics production step after energy equilibration and subsequently compared with published X-ray structural data for MC-LR (PDB ID: 1EVA) [42]. Of the 72 MC-LR bonds monitored, a total of $65(93 \%)$ were within one to two standard deviation values of their experimentally-determined values; of the 98 angles monitored, a total of $92(95 \%)$ were within one to two standard deviation values of the experimental values (42) (SI, Appendix B). Because no experimental structural data were available for the MC-LA structure, the accurate simulation of the configuration of MC-LR was considered evidence that COMPASS was a valid force field for simulating other MC variants.

\subsection{Molecular Modeling Simulations}

The model MONT surface $[26,29]$ was provided by a periodically replicated lamellum of Wyoming-type MONT, dimensioned at $21.12 \AA x 18.98 \AA$ in the $x y$ plane, with stoichiometry of $X_{0.5}\left(\mathrm{Si}_{8}\right)\left(\mathrm{Al}_{3.5} \mathrm{Mg}_{0.5}\right) \mathrm{O}_{20}(\mathrm{OH})_{4}-X$ was satisfied by the electrolytes and/or the MC compound. The total charge in the simulated cell was -4 resulting from random isomorphic substitutions in the octahedral sites $(\mathrm{Al} \rightarrow \mathrm{Mg})$; there were no adjacent substitution sites. A cell $z$-spacing of $58 \AA$ was chosen in order for the adsorptives to be minimally affected by the periodicity of the mineral basal surface and thus primarily reflect behavior at water-mineral interfaces in bulk solution [43]. The hydrated environment above the MONT surface, confined within a layer-to-layer $z$-spacing of 58 $\AA$, consisted of a $0.1 \mathrm{M}$ ionic strength solution (at water density of $1 \mathrm{~g} \mathrm{~cm}^{-3}$ ) populated with either $\mathrm{NaCl}$ or $\mathrm{CaCl}_{2}$ electrolytes. The chosen ionic strength is to reflect highnutrient surface and sediment pore waters. Circumneutral pH conditions were simulated 
wherein the carboxylate groups are deprotonated and the amino group is protonated. The pKa values of the different ionizable amino acid residues in MC-LR were previously determined (44): $\mathrm{pKa}<2.19$ for the carboxylates groups of the methyl-Asp and D-Glu residues and $\mathrm{pK}_{\mathrm{a}}>12.48$ for the protonated amino group of the Arg residue.

The MC compound and MONT surface were charge-balanced according to the electrolytes in the solution of the simulated system. For MONT, Na4-MONT or [Ca4$\mathrm{MONT}_{\mathrm{Cl}}{ }_{4}$ systems were created, respectively, for the simulated system with $\mathrm{NaCl}$ or $\mathrm{CaCl}_{2}$ solution. The two MC variants were different in their charge distribution: MC-LR contained two negative charges from the carboxylate moieties from the methyl-Asp and D-Glu residues and one positively-charged Arg residue; MC-LA only had the two negative charges from the two carboxylate moieties (Figures $1 \mathrm{~A}$ and 1B). Therefore, MC-LR had a total charge of -1 , and MC-LA had a total charge of -2 . Charge-neutral MC-LR used in the simulation systems was MC-LR-Na or [MC-LR-Ca]Cl and the charge-neutral MC-LA was MC-LA-Na2 or [MC-LA-Ca2]Cl2. The charge-neutral MC variants were subjected to a series of energy and geometry optimizations as delineated above.

A search for the optimal configurations of the adsorbate structures was conducted by using an annealing Monte Carlo approach [36] as implemented in the Adsorption Locator Module. This approach was previously demonstrated to generate configurations of adsorbate structures of antibiotic-MONT systems, which were appropriate to simulate X-ray diffraction profiles in good agreement with experimentally-obtained X-ray diffraction data $(30,32)$. In this Monte Carlo approach, the Adsorption Locator simultaneously subjected all the adsorptives (i.e. electrolytes, MC, and waters) to both 
translation and rotation steps with respect to the MONT basal surface over a temperature cycle from $300 \mathrm{~K}$ to $600 \mathrm{~K}$, repeated five times, while simultaneously monitoring interaction energies. This Monte Carlo search generated a set of the 10 best configurations of geometrically-optimized and energy-minimized arrangement of the adsorbate structures. The lowest-energy configuration from these Monte Carlo results, which was subsequently subjected to a molecular dynamics relaxation step which was carried out in a canonical NVT ensemble (fixed number of atoms, simulation cell volume, and constant temperature) for $500-1000 \mathrm{ps}$ ( $1 \mathrm{fs}$ time step) at $298 \mathrm{~K}$. The final adsorbate structures for MC-LR and MC-LA in $\mathrm{NaCl}$ or $\mathrm{CaCl}_{2}$ are illustrated in Figures 2A-2E.

\subsection{Analysis of Adsorbate Structures}

The final thermodynamically-favorable adsorbate structures obtained from the combined Monte Carlo-molecular dynamics algorithms were analyzed for the following:

(1) MC conformation in the different aqueous solutions and (2) binding interactions of $M C$ at the water-mineral interface.

In addition to the Adda group, the two MC variants both contain two charged amino acids (D-Glu and methyl-Asp) and three hydrophobic amino acids (Ala, Leu, and methyl-dehydro-Ala) in their cyclic peptide (Figure 1). In addition, MC-LR is distinguished by the presence of the positively-charged Arg residue whereas MC-LA has an additional hydrophobic Ala amino acid residue (Figure 1). The Discovery Studio software package [29] was used to create surface maps of hydrophilic and hydrophobic regions in each $\mathrm{MC}$ variant to capture changes in the conformation; the distribution of 
these regions in response to the different aqueous environments were monitored. Metal cation complexation by the MC compound was monitored by capturing coordinated atoms present within the solvated shell radius of $\mathrm{Na}^{+}$or $\mathrm{Ca}^{2+}(\leq 2.6 \AA)$. Hydrogenbonding interactions were assessed with a maximum $\mathrm{H}$-acceptor distance of $2.5 \AA$ and a minimum angle of $90^{\circ}$ between donor- $\mathrm{H}-$ acceptor [34].

\section{Results}

\subsection{Different MC Conformations and Metal Complexation}

For all the $\mathrm{MC}$ conformations, a high-hydrophobicity region in the $\mathrm{MC}$ molecule corresponded to the localization of Leu and a high-hydrophilicity region was associated with D-Glu and/or Arg in the peptide ring (Figures 2E-2H). However, the hydrophobic alkyl chain in Adda and the hydrophilic carboxylate moiety in methyl-Asp were not shown accordingly by the surface maps and were shown instead to be midway on the hydrophilicity-hydrophobicity spectrum, thus equally hydrophobic and hydrophilic (Figures $2 \mathrm{E}-2 \mathrm{H}$ ). In the case of the Adda chain, the slightly polar methoxy moiety dampened the hydrophobicity of the alkyl chain and aromatic ring of the Adda arm whereas the hydrophobic methyl group attached to methyl-Asp counteracted the hydrophilic character of the carboxylate moiety in Asp (Figures 2E-2H). The extent of the distribution of the hydrophilic and hydrophobic regions across the MC compound captured changes in MC conformations in the different simulated systems (Figures 2E$2 \mathrm{H})$. We found that specific conformations were promoted by both intramolecular interactions within the $\mathrm{MC}$ compound and the type of metal complexation by the $\mathrm{MC}$ ligands (Figures 3 and 4). 
In the MC-LR-Na, a near perpendicular bending of the Adda group towards the cyclic peptide was accompanied by a globular-like shape of the peptide ring, a conformation that limited the exposure of the hydrophobic region from the Leu residue (Figure 2E). Several intramolecular interactions contributed to the MC-LR conformation in the $\mathrm{NaCl}$ solution (Figures 3A and 3B). First, only the carboxylate $\mathrm{O}$ atoms of D-Glu coordinated $\mathrm{Na}$ from the solution whereas the carboxylate from the methyl-Asp was chargebalanced by the neighboring positively-charged guanidinium group in Arg (Figure 3A). Second, there were three intramolecular $\mathrm{H}$-bonds in the MC-LR-Na: one $\mathrm{H}$-bond between the carbonyl and amino groups located within the Adda group close to its attachment to the peptide ring, one $\mathrm{H}$-bond between the amino groups of two neighboring amino acids (Ala and Leu), and one $\mathrm{H}$-bond between one of the guanidinium $\mathrm{N}$ atoms and the terminal amino of the Arg residue linking Arg to the peptide chain (Figure 3B). Given the localization of methyl-Asp and Arg residues in MCLR (Figure 1A), the electrostatic pairing between these two residues facilitated a compressed conformation of MC-LR peptide chain (Figure 3A). In contrast to the MCLR conformation in $\mathrm{NaCl}$, the MC-LA in $\mathrm{NaCl}$ had a more openly elongated conformation of the cyclic peptide chain (Figures 2E and 2F). The MC-LA-Na had its hydrophobic Leu residue more exposed to the solvent than in the MC-LR-Na compound (Figures 2E and 2F). Furthermore, the MC-LA-Na conformation did not facilitate any intramolecular $\mathrm{H}$-bonds (Figures 3C and 3D). Both carboxylate ligands (from methylAsp and from D-Glu) in the peptide ring of MC-LA-Na were charged balanced by $\mathrm{Na}^{+}$ ions. Figure $3 \mathrm{C}$ illustrates specifically the coordination of a $\mathrm{Na}^{+}$ion by the D-Glu in $\mathrm{MC}$ $\mathrm{LA}$, a residue that was discussed previously to be involved in intramolecular 
electrostatic interaction within MC-LR-Na (Figure 3A). The lack of an Arg residue in MC-LA excluded the occurrence of an Arg-mediated intramolecular interaction in MCLA.

With respect to the simulated systems with $\mathrm{CaCl}_{2}$ solution, it was clear that the resulting $\mathrm{MC}$ conformations were imposed by the configuration of the Adda arm combined with the mode of $\mathrm{Ca}^{2+}$ complexation by carboxylate ligands in the $\mathrm{MC}$ peptide ring (Figures 2G, 2H, 4A-4D). By comparison to the MC-LR-Na-MONT system, the MC-LR in the MC-LR-Ca-MONT had a peptide ring in a more extended configuration (Figure 2G, 4A, 4B). However, the degree of the bending angle of the aromatic ring in the Adda arm in MC-LR-Ca was more severe than the perpendicular bent exhibited by the Adda arm in MC-LR-Na described above (Figures 3A, 4A). We also observed a higher exposure of hydrophilic region of the positively-charged Arg residue in MC-LRCa than MC-LR-Na (Figures (2E and $2 \mathrm{G}$ ). The latter difference in the hydrophilic character of the MC-LR conformation was attributed to the Ca complexation by both carboxylate ligands, coming each from D-Glu and methyl-Asp localized on opposite ends of the peptide ring (Figure 4A). Therefore, no carboxylate moiety in MC-LR-Ca was available to participate in intramolecular electrostatic interaction with the Arg residue, which was in turn left to be more exposed in the solution of the MC-LR-CaMONT system than in the MC-LR-Na-MONT system (Figures 2G and 4A). In contrast to MC-LR-Ca, MC-LA-Ca had an overall more open conformation of both the Adda arm and the peptide ring (Figures 2H, 4C, 4D). The Adda arm was fully extended in MC-LACa due to the lack of bending of the Adda arm towards the peptide ring as observed in the previous $\mathrm{MC}$ configurations (Figures $2 \mathrm{E}-2 \mathrm{H}$ ). In terms of the structural arrangement 
of the peptide ring, the vicinal localization of the two carboxylate moieties in MC-LR-Ca was contrasted in the MC-LA-Ca because each carboxylate ligand individually chelated a $\mathrm{Ca}^{2+}$, resulting in a more open structure of the peptide ring in MC-LA-Ca than in MCLR-Na (Figures 4). Both MC-LR-Ca and MC-LA-Ca structures exhibited two intramolecular $\mathrm{H}$-bonds but, due to the conformational differences, the amino acid resides involved in these interactions were different (Figures 4B and 4D). The two intramolecular H-bonds in MC-LR-Ca were the same as the first two listed in description of the MC-LR-Na system above whereas the two intramolecular H-bonds in MC-LA-Na occurred between non-neighboring residues (Figures 4B and 4D).

In sum, the MC conformational arrangement was due to the bending angles of the Adda arm coupled with metal complexation and intramolecular interactions by the peptide ring. The resulting conformation of the $M C$ variants in turn dictated the exposure of hydrophobic and hydrophilic residues of the MC variants. Next, we investigated how these different conformations mediated the following mechanisms of interactions at the water-mineral interface: electrostatic, cation-bridged, and $\mathrm{H}$-bonding interactions

\subsection{Direct Electrostatic and Cation-Bridged Interactions with MONT}

As mentioned in the Introduction, the protonated guanidinium in the Arg residue of MC variants is considered important for facilitating adsorption on the clay minerals [15]. We found that in the MC-LR-Ca-MONT system, the protonated guanidinium moiety of the Arg residue was $3.5 \AA$ from the clay surface, above a negatively-charged region arising from an isomorphic substitution of $\mathrm{Mg}$ for $\mathrm{Al}$ in the octahedral sheet (Figure $5 \mathrm{~A}$ ). By contrast, the MC-LR in MC-LR-Na-MONT system did not participate in any 
electrostatic interaction with the clay surface due to the intramolecular electrostatic interaction in MC-LR-Na as described above. Accordingly, the optimized adsorbate structures illustrate that MC-LR-Na was suspended in the solution whereas MC-LR-Ca was shown adsorbed at the mineral surface (Figures $2 \mathrm{~A}$ and $2 \mathrm{C}$ ). The complexation of $\mathrm{Ca}^{2+}$ by two carboxylate ligands in the peptide ring of MC-LR-Ca therefore liberated up the Arg residue for involvement in electrostatic interactions with the clay surface (Figures 3B and 5A).

In contrast to MC-LR, MC-LA does not possess a protonated Arg residue to mediate electrostatic attraction with the clay surface (Figure 1).However, because the $\mathrm{Ca}$ ion in MC-LA-Ca was coordinated by only one carboxylate ligand (from D-Glu), the chelated Ca was able to facilitate a cation-bridged interaction with the negatively-charged clay surface (Figure 5B). However, the Ca-bridged interaction is an outer-sphere complex mediated by water molecules that coordinate simultaneously the clay surface and the Ca ion. The D-Glu carboxyl O atoms were at a distance of 2.03-2.06 $\AA$ from $\mathrm{Ca}^{2+}$, which was also solvated by water $\mathrm{O}$ atoms at a distance of $2.32-2.50 \AA$ (Figure $5 \mathrm{~B}$ ). The Casolvated waters were involved in $\mathrm{H}$-bonding interactions with the mineral surface, thus indicating that MC-LR-Ca-MONT ternary complex was an outer-sphere (i.e. waterbridged) complex [45, 46] (Figure 5B).

\subsection{Water-Bridged H-bonding Interactions with MONT}

Previous spectroscopic [27] and molecular dynamics simulation [47, 48] studies with polar organic contaminants such as herbicides and antibiotics, which contain both amino and carboxylate groups, have reported the role of both direct and water-bridged 
$\mathrm{H}$-bonds between these organic compounds and MONT. Direct H-bonds between the MCs and the MONT surface were not found in any of our MC-MONT structures. However, we found that several water-bridging $\mathrm{H}$-bonding interactions stabilized the favorable adsorbate structures of the MC-MONT systems.

As mentioned in the previous section, water molecules $\mathrm{H}$-bonded to the mineral surface participated in the outer-sphere complexation of $\mathrm{Ca}^{2+}$ coordinated by MC-LA (Figure 5B). Moreover, we found that these latter water molecules were further stabilized by vicinal water molecules, which were $\mathrm{H}$-bonded both to the Ca-solvated waters and the mineral surface (Figure 5B). Therefore, a network of water-bridged interactions mediated stable adsorbate structures of MC-LA on the mineral surface.

Further examination of both MC-LR-MONT systems also revealed the occurrence of other water bridged interactions (Figures $5 \mathrm{C}$ and $5 \mathrm{D}$ ). Although the Arg residue in MCLR-Na was involved in intramolecular interaction with the methyl-Asp residue (Figure 3B), the Arg residue still participated in water-bridged interactions with the MONT surface (Figure 5C). Specifically, water molecules connected the $\mathrm{H}$ atoms of the guanidinium group to the clay via a chain of intermolecular $\mathrm{H}$-bonds (Figure $5 \mathrm{C}$ ). The MC-LR-Ca-MONT system also exhibited multiple water-bridged interactions involving both the guanidinium group of the Arg residue and the carbonyl group of D-Ala (Figure 5D).

\section{Discussion}

\subsection{Mechanistic Insights into MC Conformations Deduced from Experiments}


A previous study [25] suggested that the presence of monovalent cations can lead to a compressed configuration of MC-LR in solution. This change in conformation was proposed to be a result of a reduction in the intramolecular charge repulsion induced by the cations. It was also reported that Ca can change the configuration of MC-LR, but the nature of this structural change was not specified [25]. Our molecular modeling results indicated that multiple intramolecular H-bonds facilitated a globular MC-LR conformation in the $\mathrm{NaCl}$ solution (Figures $2 \mathrm{~A}, 2 \mathrm{E}, 3 \mathrm{~A}$, and $3 \mathrm{~B}$ ). This conformation was due to the intramolecular electrostatic attraction between the guanidinium moiety of the Arg residue at one side of the peptide ring and the carboxylate residue of the D-Glu on the other side (Figures 3A and 3B). Conformational differences between MC-LR-Na and MC-LR-Ca were also captured by our simulation results (Figures 3 and 4). Notably, complexation of $\mathrm{Ca}$ by both carboxylate moieties in the peptide cycle led to the availability of the Arg residue to be involved in intermolecular interactions (Figures $4 \mathrm{~A}$ and $5 \mathrm{~A})$.

Our results also revealed that the peptide chemistry of the MC variant influenced the conformation adopted by the MC compound (Figures 3 and Figure 4). For instance, the simulated system of MC-LA in $\mathrm{NaCl}$ had an open conformation of the peptide ring, due to the lack of the positively-charged Arg residue present in MC-LR. Therefore there was no intramolecular electrostatic interaction possible within MC-LA. Our simulated configurations of MC-LA in $\mathrm{CaCl}_{2}$ also exhibited an open conformation because the two carboxylate ligands were not chelated to the same $\mathrm{Ca}$ ion as in the MC-LR-Ca (Figures 4a and 4B). However, we envision that MC-LA-Ca could take on a more compressed conformation in a scenario wherein both carboxylates were coordinating the same $\mathrm{Ca}^{2+}$. 


\subsection{Mechanistic Insights into Previous Experimental Adsorption Studies}

An extraction study [13] of sediments spiked with MC-LR and MC-RR reported higher recovery of MC-LR than of MC-LR and thus put forth the hypothesis that Arg residues mediate higher adsorption to sediments. In agreement with this hypothesis, two other studies $[14,15]$ have also indicated higher adsorption of MC-RR to sediments than MC$\mathrm{LR}$, further stressing the importance of the Arg residue in mediating adsorption. In our simulated systems, the MC-LR-Ca-MONT system demonstrated direct electrostatic interaction with the clay surface through the protonated Arg residue, which was further stabilized by water-bridged interactions. In contrast, the MC-LR-Na-MONT system lacked this electrostatic interaction due to the conformation of the MC-LR, which prevented the free movement of the Arg side chain. Our results thus implied that favorable conformations of $\mathrm{MC}$ variants at the water-mineral interface are requisite to mediating mechanisms of adsorption on MONT.

We captured cation-bridged and different types of $\mathrm{H}$-bonding interactions between the MC variants and the MONT surface, albeit there was a dependence both on the MC variant and its conformation. Although MC-LA does not possess a positively-charged Arg residue, the MC-LA was bridged to the clay surface via a chelated $\mathrm{Ca}^{2+}$ (Figure 5B). This ternary MC-LA-Ca-MONT complex invoked the role of water bridges in facilitating a stable outer-sphere complex (Figure 5E). In agreement with our finding, a previous molecular modeling study [26] of the adsorption of the herbicide 2,4dichlorophenoxyacetic acid on Ca-MONT reported that an outer sphere complex was more thermodynamically stable than the inner sphere complex. In addition, we found 
multiple water-bridged interactions were also important to stabilize the favorable MCMONT adsorbate structures in the other simulated scenarios (Figures 5C and 5D).

\subsection{Concluding Remarks}

In the introduction, building on findings from previous macroscopic studies, we put forth four hypotheses regarding the mechanisms of $\mathrm{MC}$ adsorption at the water-mineral interfaces within soil particles in contaminated waters and sediments: (1) MC conformation is induced by aquatic chemistry, (2) specific MC conformation is required for adsorption, and (3) $\mathrm{H}$-bonding and cation-bridging interactions are important in the absence of electrostatic interactions. In support of the first two hypotheses, our simulated adsorbate structures indicated that conformational arrangements of different $M C$ variants in solution guided the specific mechanisms of $M C$ adsorption at the waterMONT interfaces. While electrostatic interaction was proposed as the main adsorption mechanism, our adsorbates demonstrated that multiple water-bridged $\mathrm{H}$-bonds play an important role in stabilizing MC-mineral complexes, in agreement with the third hypothesis. And additional participation of multivalent cation-bridging was specific to the chemistry of the MC variant.

The relevance of our modeling predictions for environmental fate assessment should be considered with respect to four important factors. First, the scope of our analysis focused on the most thermodynamically-favorable configurations under our simulated conditions. Interaction of $\mathrm{MC}$ variants at water-mineral interfaces is a dynamic process involving a variety of adsorbate structures. We acknowledge that other favorable configurations could also occur simultaneously in contaminated environments. Second, 
we show that the type of $\mathrm{MC}$ complexation of two metal cations commonly found in environmental matrices can re-structure the arrangement of the hydrophilic and hydrophobic moieties of the $M C$ and thus influence binding interactions to mineral particles. It should also be noted that natural waters and soils contain an array of electrolytes beyond the composition of our simulated solutions that likely can influence MC conformation. In addition, the presence of organic matter in natural clay sediments can influence MC retention. Experimental adsorption results from a previous study [15] proposed an organic matter content-dependent effects on MC complexation by clay minerals. Decreased MC adsorption in the presence of low organic matter content suggested competition with MC compounds for adsorption sites on clay minerals [15]. However, when organic matter content was relatively high, MC adsorption was enhanced, implying promoted retention of $\mathrm{MC}$ within the organo-mineral matrix. We proposed that the water-bridged $\mathrm{H}$-bonds and metal complexes predicted here for MCmineral adsorbate structures may also facilitate the trapping of MCs within natural organic matter that coats clay mineral surfaces. Follow-up molecular modeling studies can elucidate further the interplay between the molecular interactions within MC-clay complexes and those within MC-organic-matter-clay complexes using organic matter of varying chemistry and structure. Finally, experimental spectroscopic investigation is warranted to confirm the adsorption mechanisms of MC variants to different soil particles. Here, as a prologue to these spectroscopic studies, we employed a computational approach to obtain molecular insights into the mechanisms of interactions of two MC variants with a clay mineral under different aqueous scenarios. In this respect, our modeling predictions advance a mechanistic framework for considering 
$\mathrm{MC}$ chemistry in concert with aquatic chemistry in the removal of MCs from contaminated waters and sediments by clay and organo-clay particles.

\section{Supplementary Information}

Appendix A: Root-mean-square-deviation (RMSD) of MC-LR

Appendix B: Modeling validation of microcystin-LR.

\section{Acknowledgements}

A.L.P. acknowledges a Cornell University Graduate School Fellowship. T.G.A.'s undergraduate research was supported by a grant from the U. S. National Science Foundation (Division of Earth Sciences, EAR 1343063). This work was funded by a start-up package from Cornell University.

\section{References}

1. Carmichael WW. 1992. Cyanobacteria secondary metabolites- the cyanotoxins. J. Appl. Microbiol. 72, 445-459.

2. Davidson K, Tett P, Gowen R. 2011. Harmful Algal Blooms. In Hester RE, Harrison RM, eds, Marine Pollution and Human Health, Royal Society of Chemistry Publishing, Oxford, England, 95-127.

3. Chen W, Song L, Peng L, Wan N, Zhang X, Gan N. 2008 Reduction in microcystin concentrations in large and shallow lakes: Water and sedimentinterface contributions. Water Res., 42, 763-773.

4. Ross C, Santiago-Vázquez L, Paul V. 2006. Toxin release in response to 
oxidative stress and programmed cell death in the cyanobacterium Microcystis aeruginosa. Aquat. Toxicol. 78, 66-73.

5. Backer LC, McNeel SV, Barber T, Kirkpatrick B, Williams C, Irvin M, Zhou Y, Johnson TB, Nierenberg K, Aubel M, LePrell R, Chapman A, Foss A, Corum S, Hill VR, Kieszak SM, Cheng Y-S. 2010. Recreational exposure to microcystins during algal blooms in two California lakes. Toxicon, 55, 909-921.

6. Malbrouck C., Kestemont P. 2006. Effects of microcystins on fish. Environ. Tox. Chem. 1, 72-86.

7. Gupta N, Pant SC, Vijayaraghavan R, Rao PV. 2003. Comparative toxicity evaluation of cyanobacterial cyclic peptide toxin microcystin variants (LR, RR, YR) in mice. Toxicology 188, 285-296.

8. Tsuji K, Asakawa M, Anzai Y, Sumino T, Harada K-I. 2006. Degradation of microcystins using immobilized microorganism isolated in an eutrophic lake. Chemosphere 65, 117-124.

9. Song H, Reichwaldt ES, Ghadouani A. 2014. Contribution of sediments in the removal of microcystin-LR from water. Toxicon 83, 84-90.

10. Feitz AJ, Waite TD, Jones GJ, Boyden BH, Orr PT. 1999. Photocatalytic degradation of the blue green algal toxin microcystin-LR in a natural organicaqueous matrix. Environ. Sci. Technol. 33, 243-249.

11. Woermer L, Huerta-Fontela M, Cires S, Carrasco D. 2010. Quesada, A. Natural photodegradation of the cyanobacterial toxins microcystin and cylindrospermopsin. Environ. Sci. Technol. 44, 3002-3007.

12. Morris RJ, Williams DE, Luu HA, Holmes C, Andersen RJ, Calvert SE. 2000. The 
adsorption of microcystin-LR by natural clay particles. Toxicon 38, 303-308.

13. Tsuji K, Masui H, Uemura H, Mori Y, Harada K. 2001. Analysis of microcystins in sediments using MMPB method. Toxicon 39, 687-692.

14. Grützmacher G, Wessel G, Klitzke S, Chorus I. 2010. Microcystin elimination during sediment contact. Environ. Sci. Technol. 44, 657-662.

15. Wu X, Xiao B, Li R, Wang C, Huang J, Wang Z. 2011. Mechanisms and factors affecting sorption of microcystins onto natural sediments. Environ. Sci. Technol. 45, 2641-2647.

16. Maghsoudi E, Prévost M, Duy SV, Sauvé S, Dorner S. 2015. Adsorption characteristics of multiple microcystins and cylindrospermopsin on sediment: Implications for toxin monitoring and drinking water treatment. Toxicon 103, 4854.

17. Miller MJ, Critchley MM, Hutson J, Fallowfield HJ. 2001. The adsorption of cyanobacterial hepatotoxins from water onto soil during batch experiments. Water Res. 35, 1461-1468.

18. Jones GJ, Orr PT. 1994. Release and Degradation of Microcystin Following Algicide Treatment of a Microcystis-Aeruginosa Bloom in a Recreational Lake, as Determined by HPLC and Protein Phosphatase Inhibition Assay. Water Res. 28, 871-876.

19. Daly RI, Ho L, Brookes JD. 2007. Effect of chlorination on Microcystis aeruginosa cell integrity and subsequent microcystin release and degradation. Environ. Sci. Technol. 41, 4447-4453.

20.Pan G, Zhang MM, Chen H, Zou H, Yan H. 2006. Removal of cyanobacterial 
blooms in Taihu Lake using local soils. I. Equilibrium and kinetic screening on the flocculation of Microcystis aeruginosa using commercially available clays and minerals. Environ. Pollut. 141, 195-200.

21. Teng W, Wu Z, Feng D, Fan J, Wang J, Wei H, Song M, Zhao D. 2013. Rapid and Efficient Removal of Microcystins by Ordered Mesoporous Silica. Environ. Sci. Technol. 47, 8633-8641.

22. Changa S-C, Li C-H, Lin J-J, Li Y-H, Lee M-R. 2014. Effective removal of Microcystis aeruginosa and microcystin-LR using nanosilicate platelets. Chemosphere 99, 59-55.

23. Sengco MR, Anderson DM. 2004. Controlling harmful algal blooms through clay flocculation. J. Eukaryot. Microbiol. 51, 169-172.

24. Campinas M, Viegas RMC, Rosa MJ. 2013. Modelling and understanding the competitive adsorption of microcystins and tannic acid. Water Res. 47, 56905699.

25. Campinas M, Rosa MJ. 2006. The ionic strength effect on microcystin and natural organic matter surrogate adsorption onto PAC. J. Colloid Interface Sci. 299, 520-529.

26. Tunega D, Gerzabek MH, Haberhauer G, Lischka H. 2007. Formation of 2,4-D complexes on montmorillonites - an ab initio molecular dynamics study. Eur. J. Soil Sci. 58, 680-691.

27. Aristilde L, Marichal C, Miéhé-Brendlé J, Lanson B, and Charlet L. 2010. Interactions of oxytetracycline with a smectite clay: A spectroscopic study with molecular simulations. Environ. Sci. Technol., 44, 7839-7845. 
28.Zhao Q, Burns SE. 2012. Molecular Dynamics Simulation of Secondary Sorption Behavior of Montmorillonite Modified by Single Chain Quaternary Ammonium Cations. Environ. Sci. Technol. 46, 3999-4007

29. Zhu R, Hu W, You Z, Ge F, Tian K 2012. Molecular dynamics simulation of TDD adsorption on organo-montmorillonite. J. Colloid Interf. Sci., 377, 328-333.

30. Aristilde L, Lanson B, Charlet L. 2013. Interstratification Patterns from the pHDependent Intercalation of a Tetracycline Antibiotic within Montmorillonite Layers. Langmuir 29, 4492-4501.

31. Liu X, Zhu R, Ma R, Ge F, Xu Y. Molecular dynamics simulation study of benzene adsorption to montmorillonite influence: Influence of the hydration status. Colloids Surf A: Physiochem. Eng. Aspects 434, 200-206.

32. Aristilde L, Lanson B, Miéhé-Brendlé J., Marichal C., Charlet L. 2016. Enhanced interlayer trapping of a tetracycline antibiotic within montmorillonite layers in the presence of $\mathrm{Ca}$ and Mg. J. Colloid Interf. Sci., 464, 153-159

33. Sun, H.1998. COMPASS: An ab initio force-field optimized for condensed-phase applications overview with details on alkane and benzene compounds. J. Phys. Chem. B 102, 7338-7364.

34. Accelrys Software Inc. 2013. Materials Studio Modeling Environment, Release 7.0, San Diego: Accelrys Software Inc.

35. Berendsen, HJC, Grigera JR, Straatsma TP. The missing term in effect pair potentials. 1987. J. Phys. Chem. 91, 6269-6271.

36. Cygan RT, Liang J-J, Kalinichev AG. Molecular models of hydroxide, oxyhydroxide, and clay phases and the development of a general force field. 
2004. J. Phys. Chem. B 108, 1255-126

37. McQuaid MJ, Sun H, Rigby D. 2004. Development and validation of COMPASS force field parameters for molecules with aliphatic azide chains. J. Comput. Chem. 25, 61-71.

38. Aristilde L, Sposito G. 2008. Molecular modeling of metal complexation by a fluoroquinolone antibiotic. Environ. Toxicol. Chem. 27: 2304-2310

39. Sutton R, Sposito G, Diallo MS, Schulten H-R. 2005. Molecular simulation of a model of dissolved organic matter. Environ. Toxicol. Chem. 24, 1902-1911.

40. Aristilde, L.; Sposito, G. 2010. Binding of ciprofloxacin by humic substances: a molecular dynamics study. Environ. Toxicol. Chem. 29, 90-98.

41. Sutton R, Sposito, G. 2006. Molecular simulation of humic substance-Camontmorillonite complexes. Geochim. Cosmochim. Acta 70, 3566-3581.

42. Bagu JR, Sönnichsen FD, Williams D, Andersen RJ, Sykes BD, Holmes CF. 1995. Comparison of the solution structures of microycstin-LR and motuporin. Nat. Struct. Biol. 2, 114-116.

43. Bourg IC, Sposito G. 2011. Molecular dynamics simulations of the electrical double layer on smectite surfaces contacting concentrated mixed electrolyte ( $\mathrm{NaCl}-\mathrm{CaCl} 2)$ solutions. J Colloid. Interface Sci. 360, 701-715.

44. de Maagd, P.G.-J., Hendriks, A.J., Seinen, W., Sijm, D.T.H.M., 1999.pH-dependent hydrophobicity of the cyanobacteria toxinmicrocystin-LR. Wat. Res., 33, 677-680.

45. Sposito G, Skipper NT, Sutton R, Park S-H, Soper AK, Greathouse JA. 1999. Surface geochemistry of the clay minerals. Proc. Natl. Acad. Sci. U.S.A. 96, $3358-3364$. 
46. Sposito, G. The Chemistry of Soils, 2nd ed.; Oxford University Press: USA, 2008.

47. Teppen BJ, Yu CH, Miller DM, Schäfer L. 1998. Molecular dynamics simulations of sorption of organic compounds at the clay mineral/aqueous solution interface. J. Comput. Chem. 19, 144-153.

48. Kalinichev AG, Kumar PP. 2010. Molecular dynamics computer simulations of the effects of hydrogen bonding on the properties of layered double hydroxides intercalated with organic acids. Philos. Mag. 90, 2475-2488. 


\section{Figures}

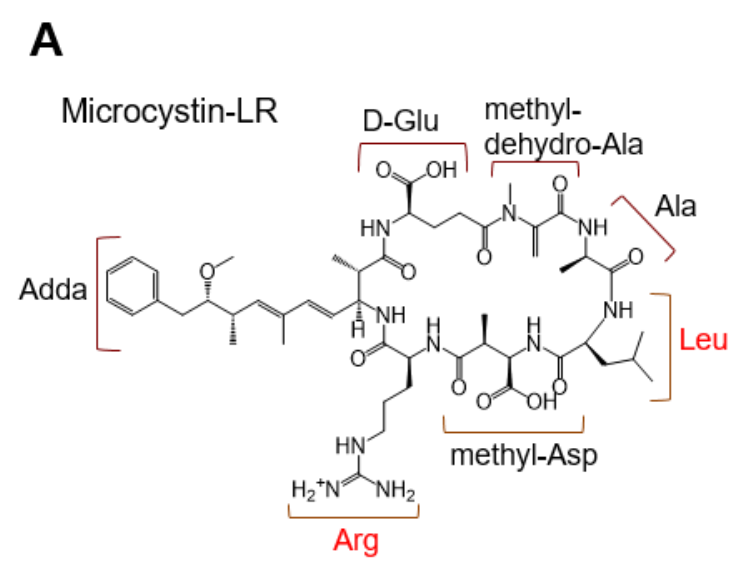

B

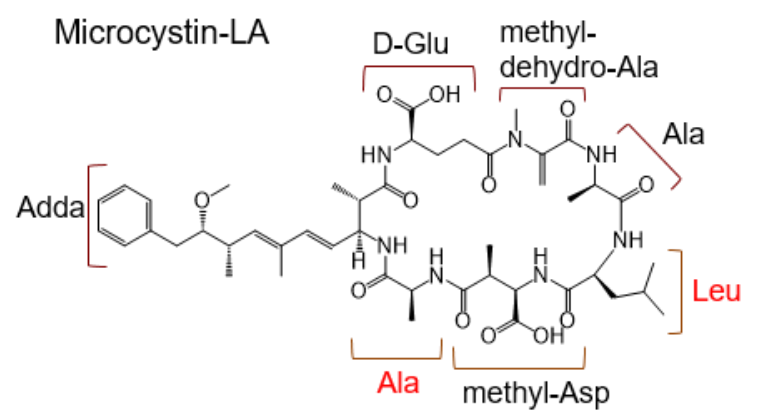

Fig. 1. Chemical structure of $(A) M C-L R$ and(B) MC-LA. Abbreviations: D-Glu (Dglutamate); methyl-dehydro-Ala (N-methyl-dehydro-alanine); Ala (alanine); Leu (leucine); methyl-Asp (methyl-aspartate); Arg (arginine); Adda (3-amino-9-methoxy2,6,8-trimethyl-10-phenyldeca-4,6-dienoic acid). 

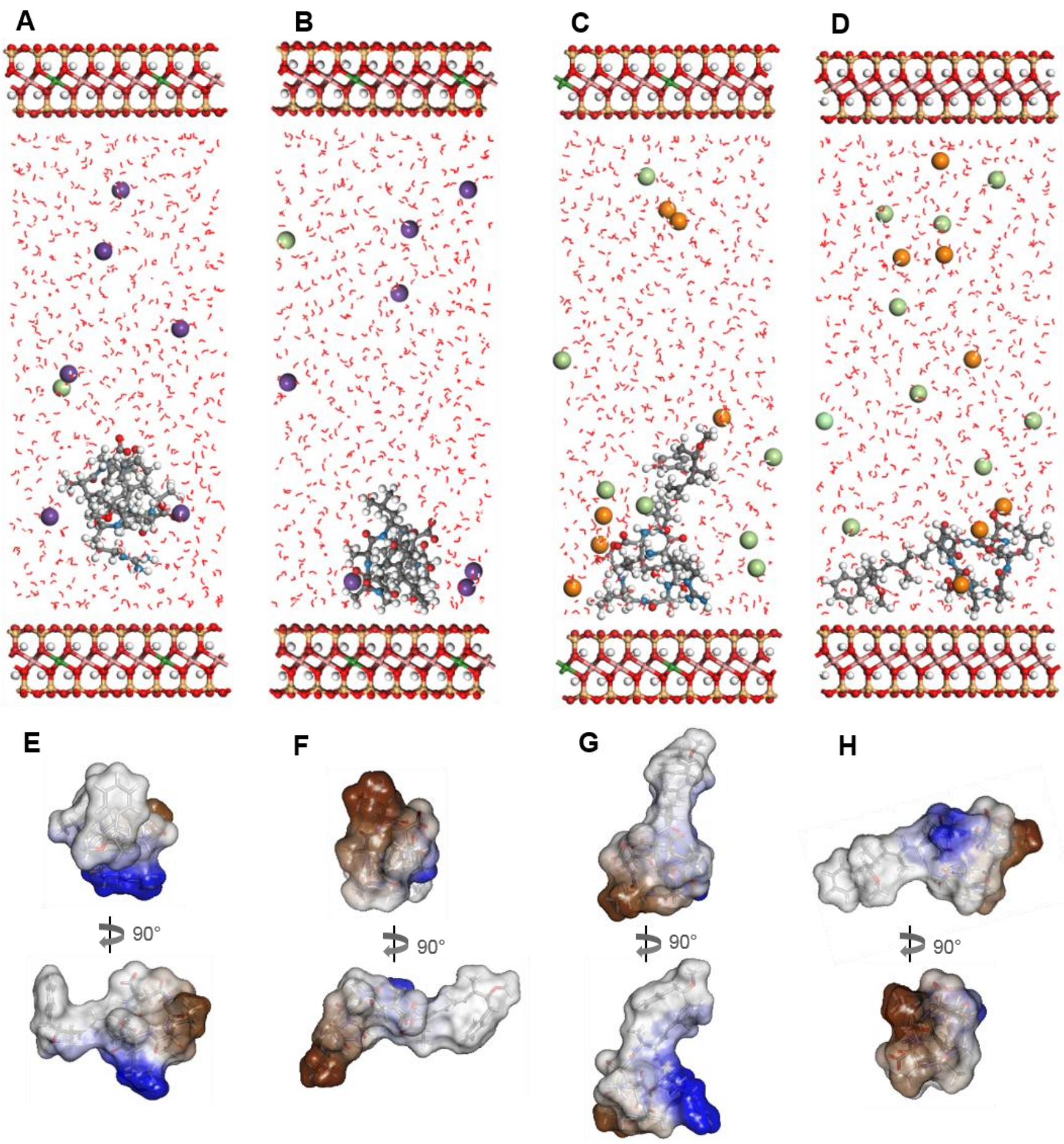

H
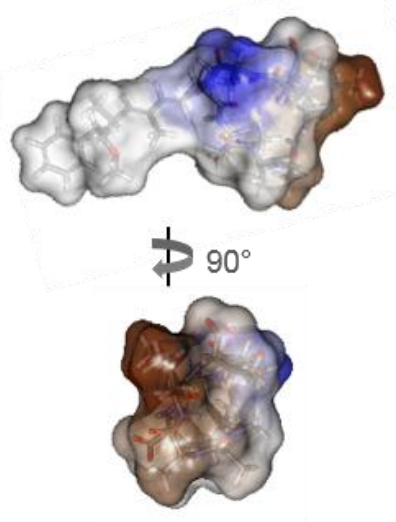

Fig 2. Configurations of adsorbate structures of (A) MC-LR-Na-MONT, (B) (B) MC-LANa-MONT, (C) MC-LR-Ca-MONT, and (D) MC-LA-Ca-MONT. Hydrophobicity maps of (E) MC-LR-Na, (F) MC-LA-Na, (G) MC-LR-Ca, and (H) MC-LA-Ca. In A-D, the top clay structure is a periodic reflection of the bottom one. Color code for A-D: red (O), gray $(\mathrm{C})$, blue $(\mathrm{N})$, white $(\mathrm{H})$, light gold $(\mathrm{Si})$, light pink $(\mathrm{Al})$, dark green $(\mathrm{Mg})$, orange $(\mathrm{Ca})$, 
purple $(\mathrm{Na})$, light green $(\mathrm{Cl})$. Hydrophobic and hydrophilic regions are shown in brown and blue, respectively, in panels E-H.
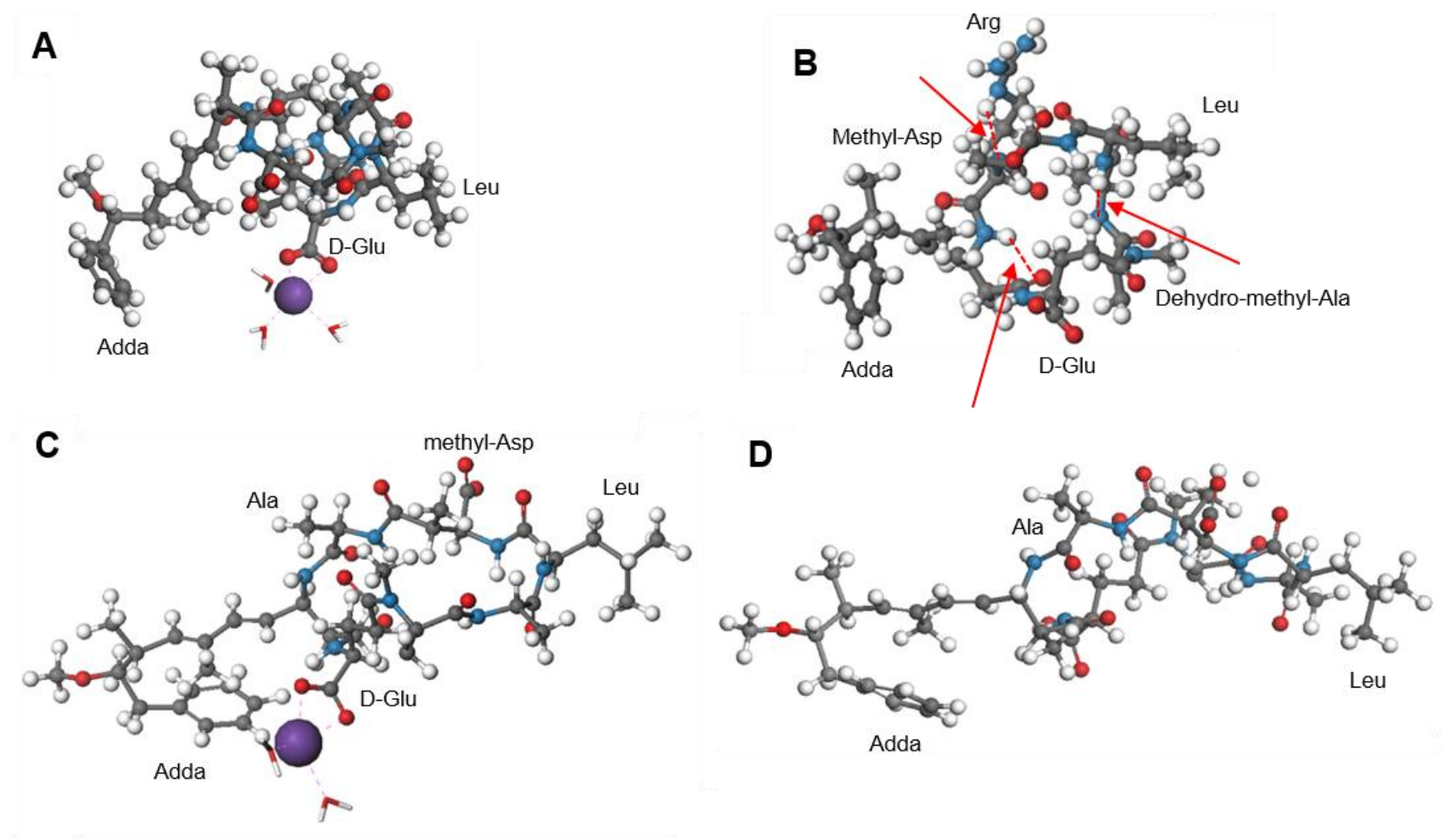

Fig 3. Close-up views of ( $A$ and $B)$ optimized $M C-L R$ in the MC-LR-Na-MONT system and ( $C$ and $D)$ optimized MC-LA in the MC-LA-Na-MONT system. Panels B and $\mathrm{D}$ show a different view of the $\mathrm{MC}$ variants to highlight the occurrence (or lack thereof) of intramolecular $\mathrm{H}$-bonds; the metal complexation was not shown in these panels for clarity. In A-D, only the waters coordinating the metal cations are shown; all other waters are removed for clarity. Atom color code: red $(O)$, gray $(C)$, blue $(N)$, white $(\mathrm{H})$, purple $(\mathrm{Na})$. Line color code: hydrogen bonds (dashed red lines indicated by the red arrows) and metal complexation (dashed pink lines lines). Atom color code: red $(\mathrm{O})$, gray $(\mathrm{C})$, blue $(\mathrm{N})$, white $(\mathrm{H})$, purple $(\mathrm{Na})$. Line color code: hydrogen bonds (red dashed lines) and metal complexation (pink dashed lines). 


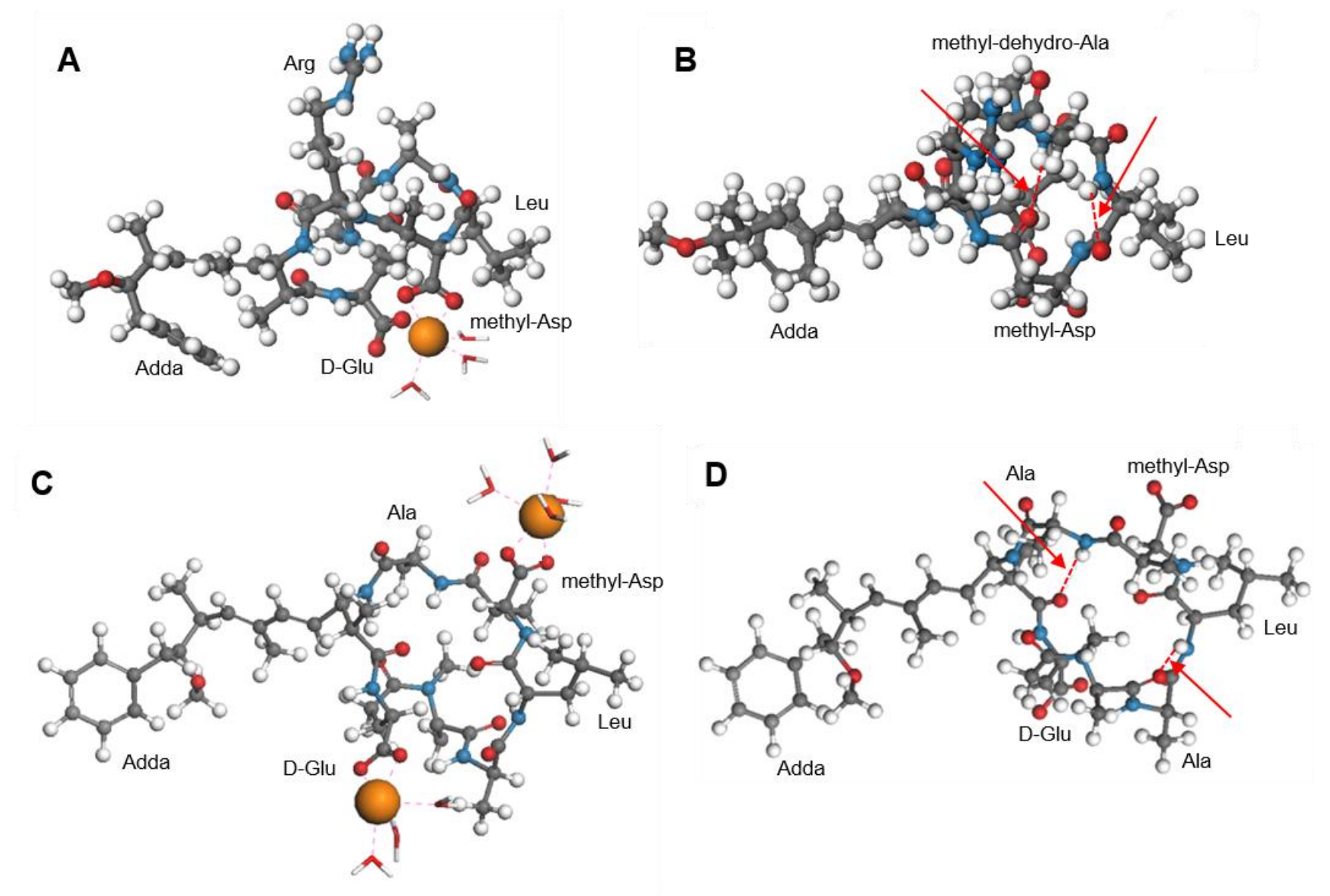

Fig. 4. Close-up views of (A and $B)$ optimized $M C-L R$ in the MC-LR-Ca-MONT system and ( $C$ and $D)$ optimized MC-LA in the MC-LA-Ca-MONT. Panels B and D show a different view of the $\mathrm{MC}$ variants o highlight the occurrence of intramolecular $\mathrm{H}$ bonds; the metal complexation was not shown in these panels for clarity. In A-D, only the waters coordinating the metal cations are shown; all other waters are removed for clarity. Atom color code: red $(\mathrm{O})$, gray $(\mathrm{C})$, blue $(\mathrm{N})$, white $(\mathrm{H})$, purple $(\mathrm{Na})$. Line color code: hydrogen bonds (dashed red lines indicated by the red arrows) and metal complexation (dashed pink lines lines). Atom color code: red $(O)$, gray $(C)$, blue $(N)$, white $(\mathrm{H})$, orange $(\mathrm{Ca})$. Line color code: hydrogen bonds (red dashed lines) and metal complexation (pink dashed lines). 
A

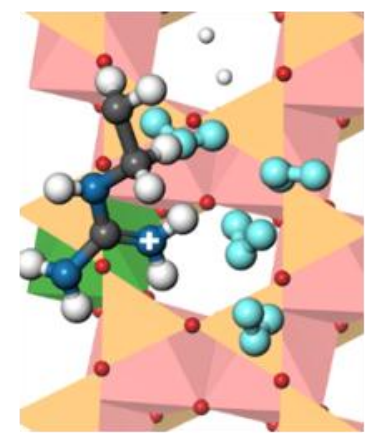

ก $180^{\circ}$

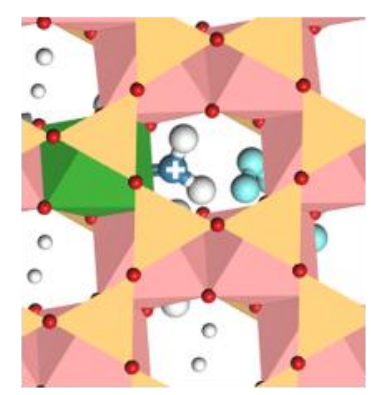

B

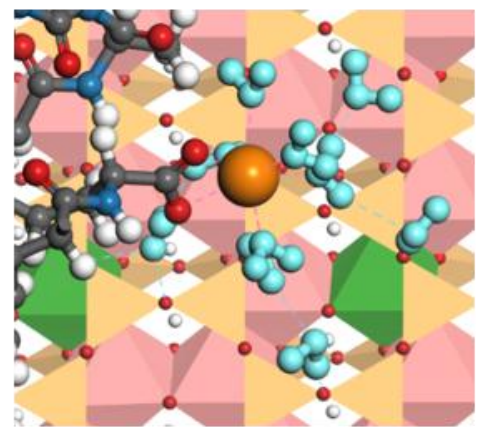

A $90^{\circ}$

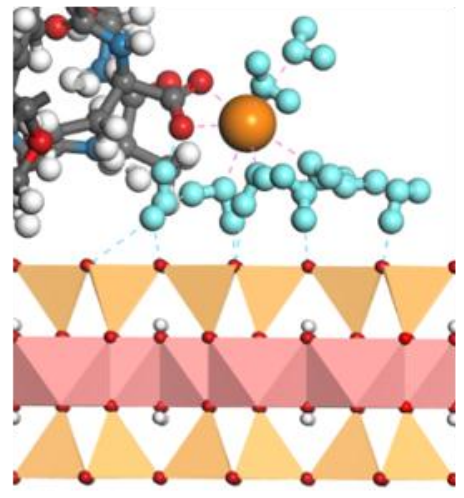

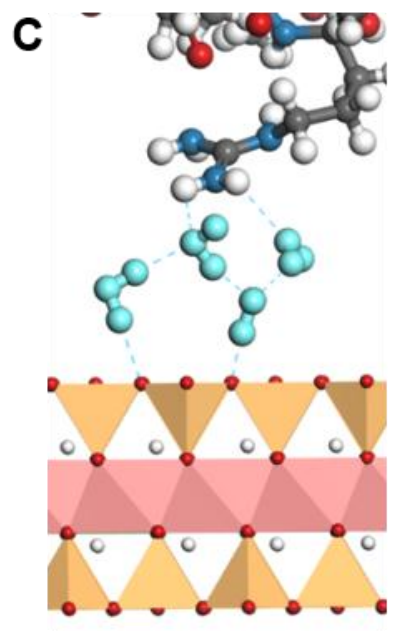

D

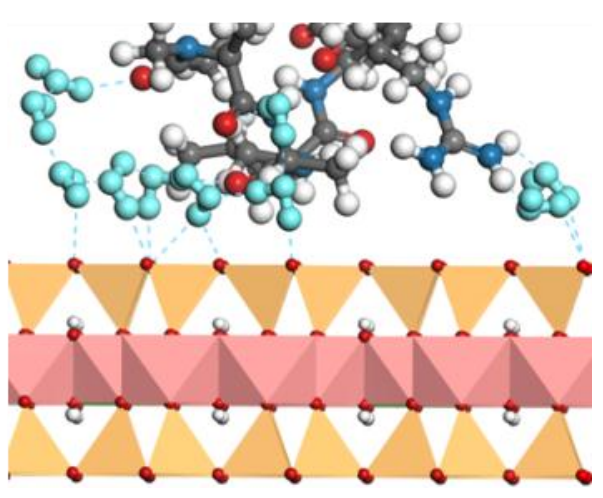

Fig 5. Molecular modeling snapshots of $(A)$ electrostatic interaction between the positively-charged guanidinium of the Arg residue and negatively charged clay in MCLR-Ca-MONT, (B) the cation-bridging interaction in MC-LA-Ca-MONT, and waterbridging interactions with the MONT surface in (C) the MC-LR-Na-MONT and (D) the MC-LR-Ca-MONT. In A and B, the rotation angle guide is from the bottom image to the top image. Color code: red $(\mathrm{O})$, gray $(\mathrm{C})$, blue $(\mathrm{N})$, white $(\mathrm{H})$, light gold $(\mathrm{Si})$, light pink (Al), dark green (Mg), water molecules (light blue). 